\title{
V13 - IN SILICO IDENTIFICATION, CLONING, EXPRESSION AND CHARACTERIZATION OF SURFACE-EXPOSED PROTEINS OF Streptococcus pneumoniae
}

$\underline{\text { Ana Paula Corrêa Argondizzo }}{ }^{1}$, Claudio Marcos Rocha de Souza ${ }^{1}$, Cristiane Pinheiro Pestana $^{1}$, Camila Borges Rodrigues ${ }^{1}$, Fabio Faria da Mota ${ }^{2}$, Ricardo Galler ${ }^{1}$, Marco Alberto Medeiros ${ }^{1}$

1 Bio-Manguinhos, Oswaldo Cruz Foundation, Technological Development, Rio de Janeiro, Brazil

2. IOC, Oswaldo Cruz Foundation, Rio de Janeiro, Brazil

Objectives: The aim of this study was to identify, in silico, surface-exposed proteins of pneumococci, clone, express and purify these recombinant proteins as well as to evaluate the expression of these proteins in bacterial cellular surface, and verify the interaction with cellular matrix proteins. The distribution of the genes in pneumococci clinical samples as well as the degree of gene and protein identity were also evaluated in this study.

Methods: In this work, we sequenced and analyzed $S$. pneumoniae serotype 5 genome and also produced a database containing pneumococcal proteins. We selected a set of genes from this database that codify to surface-exposed proteins. The cellular localization prediction was performed by in silico analysis. To confirm surface-proteins expression on the cell surface, bacterial cells were analized by FACS and by IEF. We also evaluated the ability of these proteins to interact with extracellular matrix proteins, as well as the presence and degree of identity of these genes in clinical samples by PCR and sequencing.

Results: The genes were amplified by PCR, cloned into expression vectors and the soluble proteins were purified by "IMAC". Antisera were produced by immunization of mouse with recombinant proteins adsorbed in Alhydrogel. Recombinant proteins were shown to react by immublotting with these antisera. The antisera were used to confirm the extracellular localization of the proteins in 10 serotypes of pneumococci. Among the proteins evaluated 4 were able to interact with fibronectin, laminin, fibrinogen and 
collagen IV. We confirm by PCR that the genes are present in 51 pneumococcal clinical isolates of different serotype. The degree of nucleotide and protein identity showed more than $95 \%$, by sequencing.

Conclusion: Altogether, these results strongly suggest that the proteins investigated in this study have a high degree of conservation and are present in a large number of pneumococcal serotypes as well as are expressed in bacterial cellular surface. These are important characteristics for proteins candidate to compose a recombinant vaccine. Furthermore, the interaction of some proteins with matrix proteins increase the potential of these proteins play important roles in nasal colonization. 